\title{
Pharmacology of Alzheimer's Disease: Appraisal and Prospects
}

\author{
François Sellal $^{\mathrm{a}}$ André Nieoullon ${ }^{\mathrm{b}}$ Géraldine Michel ${ }^{\mathrm{c}}$ Bernard François Michel ${ }^{\mathrm{c}}$ \\ Lucette Lacomblez $^{\mathrm{d}}$ Hugo Geerts ${ }^{\mathrm{g}}$ Alexandra Delini Stula ${ }^{\mathrm{h}}$ Régis Bordet $^{\mathrm{e}}$ \\ Danièle Bentué-Ferrer ${ }^{f}$ Hervé Allain $^{f}$ \\ ${ }^{a}$ Hôpital Universitaire, Strasbourg, ${ }^{\mathrm{b}}$ Laboratoire CNRS - LNCF Neurosciences, and ${ }^{\mathrm{c} H o ̂ p i t a u x ~ S a i n t e ~ M a r g u e r i t e ~}$ \\ et de la Conception, Marseille, ${ }^{d}$ Fédération Neurologie Mazarin/Service de Pharmacologie, Hôpital de la Pitié \\ Salpétrière, Paris, ${ }^{\mathrm{e}}$ Laboratoire de Pharmacologie, Lille, and ${ }^{\mathrm{f}}$ Laboratoire de Pharmacologie, Rennes, France; \\ ${ }^{\mathrm{g}}$ Silico Biosciences, Philadelphia, Pa., USA; ${ }^{\mathrm{h}}$ ADI International Institut, Basel, Switzerland
}

\section{Key Words}

Alzheimer's disease · Pharmacology • Innovation •

Drug $\cdot$ Methodology

\begin{abstract}
Ten years after the introduction of the first drug, tacrine, in the treatment of Alzheimer's disease, it seems appropriate to reappraise the pharmacological processes of innovation in the research field of dementia. The aim of this review is to pinpoint concrete improvements achieved in this field, regarding experimental methods and clinical evaluation of the compounds, as well as the neurochemistry of the disease and cellular targets to consider in priority. This review deals with this objective in three parts: (1) assessment of current therapeutics, (2) discussion of the experimental models and clinical practices and (3) prospective drugs of the future. The implementation of considered strategies will require the involvement and close cooperation between political decisions, pharmaceutical companies and the scientific community.
\end{abstract}

The pharmacology of Alzheimer's disease is a new field inaugurated by the marketing of tacrine in 1994. Publications on the subject are growing exponentially, with a marked mismatch between the expectations of the public and families and medical and scientific information. Drug development up to the final stages is certainly a long, expensive and complex process [1]; Alzheimer's disease is, due to a multicomposite pathophysiology, enriched by the continuing progress in neurosciences; the intellectual and financial power of the pharmaceutical industry cannot compare to the teams of large research organizations or universities; teams of doctors have become better organized in the form of networks and multidisciplinary teams and now provide unprecedented medical support for these patients, who were previously somewhat neglected. However, the ideal drug, able to eradicate this apparent epidemic observed all over the world, is still not available. The objective of this article is not to propose just another review of 'The Pharmacology of Alzheimer's Disease', but to present the conclusions of a group of experts in neurology and pharmacology in terms of a reappraisal of pharmacological research and prospects for the future development of drugs to treat dementias.

\section{KARGER \\ Fax +4161306 1234 E-Mail karger@karger.ch} www.karger.com www.karger.com/dem
Danièle Bentué-Ferrer

Department of Pharmacology, Faculté de Médecine, University of Rennes I

2, avenue du Professeur Léon-Bernard, FR-35043 Rennes (France)

Tel. +33223234713, Fax +33223234605

E-Mail daniele.bentue-ferrer@univ-rennes1.fr 


\section{Assessment of Cholinesterase Inhibitors 10 Years after Marketing}

The regulatory approval of tacrine, 10 years ago, profoundly modified the approach to Alzheimer's disease, not so much in terms of its efficacy, real but limited, but because it overcame the resignation in relation to this disease, triggering an intense research and development activity and opening the way to pharmacological management of this disease [2]. Cholinesterase inhibitors have also played an important role in terms of the clinical data that they have provided, as they have revolutionized the classification of dementias by refuting the prevailing concept of heterogeneity.

The marketing of donepezil, in 1997, marked a new step forward, by eliminating the risk of serious hepatotoxic adverse effects associated with the use of tacrine. Other drugs were subsequently launched, each providing a new pharmacological feature, resulting in continuing progress over the years: rivastigmine in 1998, followed by galantamine in 2000. Galantamine, for example, provided an additional mechanism, allosteric stimulation of nicotinic receptors [3]. In the absence of comparative clinical trials, the clinical expression of this progress can be illustrated by the progressive extension of the indications, the duration of long-term benefit, the beneficial effects on psychological and behavioural disorders and on attentional deficits. In any case, these molecules demonstrated their therapeutic value, leading to the official marketing authorisation (MA). Clinical trials have shown that cholinesterase inhibitors provided superior improvement to placebo (despite the fact that there is a lasting and marked placebo effect in this disease, probably related to the effect of management) over a period of 6 months to 1 year on major endpoints (cognition, psychological and behavioural disorders, dependence) and delayed institutionalization (maintenance of a social and family life, relief of caregiver burden), with a pharmaco-economic correlation, that is now universally accepted. Table 1 presents the main characteristics of these 4 molecules.

A review of cholinesterase inhibitors also raises the question of the other molecules which failed to reach final-stage development. In most cases, termination of the development of these molecules was due to toxic risks (for example, metrifonate and a myasthenia syndrome, xanomeline and postural hypotension), but also by drug company policy decisions.

It is also interesting to recall the history of cognitive enhancers, for which an MA has never been granted for the treatment of Alzheimer's disease, but which are still used in clinical practice. Some of these molecules have been evaluated in clinical trials demonstrating beneficial effects on cognition, but according to a questionable methodology and on poorly defined study populations, not exclusively including demented patients. These drugs were already investigating pathways other than cholinergic mechanisms. They illustrate the enormous gap between an attractive biological and pharmacological reality and their disappointing therapeutic efficacy.

The cholinergic approach can probably not provide any further progress than that achieved to date, but many questions remain unresolved and many points need to be elucidated.

1 The intensity of the response to treatment, which remains limited.

2 The concept of responders in the absence of predictive factors: this is an important issue, as the majority of patients do not obtain significant improvement of their symptoms, and the rationale for treatment and the value of continuing treatment are therefore entirely based on the results of clinical trials and the concept of stabilization by cholinesterase inhibitors compared to the natural history without treatment.

3 The marked cognitive and behavioural decline observed when treatment needs to be discontinued, explained by the high cholinesterase concentration in cholinergic synapses observed after several months of treatment with cholinesterase inhibitors. The problem of the protocol of replacement of one cholinesterase inhibitor by another, or even elaboration of a replacement treatment regimen, has not been resolved.

4 The correlation between the results of clinical trials, individual assessment of treatment in the doctor's office and their extrapolation to daily life for this population of patients, who have a poor prognosis despite treatment.

5 The possibility of a preventive, neuroprotective approach and pharmacological management of early stages called mild cognitive impairment (MCI).

6 The efficacy on dimensions other than cognition: psychological and behavioural disorders, relational problems with the family, attentional processes, executive functions.

7 The extension to other forms of dementia, particularly frontotemporal dementia, in which cholinesterase inhibitors are not indicated [4].

8 The management of severe to very severe forms, not included in the MA, which nevertheless represent a large proportion of this population. 
Table 1. Main characteristics of the four cholinesterase inhibitors currently on the market

\begin{tabular}{|c|c|c|c|c|}
\hline & $\begin{array}{l}\text { Tacrine } \\
\text { (Parke-Davis) }\end{array}$ & $\begin{array}{l}\text { Donepezil } \\
\text { (Eisai-Pfizer) }\end{array}$ & $\begin{array}{l}\text { Rivastigmine } \\
\text { (Novartis) }\end{array}$ & $\begin{array}{l}\text { Galantamine } \\
\text { (Janssen-Cilag) }\end{array}$ \\
\hline \multicolumn{5}{|l|}{ Pharmacodynamic properties } \\
\hline Mechanism of enzymatic inhibition & $\begin{array}{l}\text { non-competitive, } \\
\text { reversible }\end{array}$ & $\begin{array}{l}\text { non-competitive, } \\
\text { reversible }\end{array}$ & $\begin{array}{l}\text { non-competitive, } \\
\text { irreversible slow }\end{array}$ & $\begin{array}{l}\text { competitive, } \\
\text { reversible }\end{array}$ \\
\hline Duration of enzymatic inhibition & short & short & intermediate & short \\
\hline \multicolumn{5}{|l|}{ Pharmacokinetic properties } \\
\hline $\mathrm{C}_{\max }, \mathrm{mg} / \mathrm{l}$ & $\begin{array}{l}5.1(10 \mathrm{mg}) ; 20.7(20 \mathrm{mg}) \\
33.9(30 \mathrm{mg})\end{array}$ & $7.2(5 \mathrm{mg}) ; 25.6(10 \mathrm{mg})$ & $5.07(3 \mathrm{mg} \times 2) ; 14.1(6 \mathrm{mg} \times 2)$ & $\begin{array}{l}66(12 \mathrm{mg} \times 2) \\
137(16 \mathrm{mg} \times 2)\end{array}$ \\
\hline $\mathrm{T}_{\max }, \mathrm{h}$ & $1-2$ & $3-5$ & $0.5-2$ & $0.9-2$ \\
\hline $\mathrm{AUC}, \mu \mathrm{g} / \mathrm{l} \cdot \mathrm{h}$ & $2-4$ & 539 & $15.4(3 \mathrm{mg} \times 2) ; 55.9(6 \mathrm{mg} \times 2)$ & 1.1 \\
\hline $\mathrm{T}_{1 / 2}, \mathrm{~h}$ & $1.3-7$ & $50-80$ & $0.6-2$ & $7-8$ \\
\hline Bioavailability, $\%$ & $17-37$ & 100 & $35-40$ & 100 \\
\hline Protein binding, $\%$ & 55 & 96 & 40 & 18 \\
\hline Clearance, $1 / \mathrm{h} / \mathrm{kg}$ & 2.42 & 0.13 & $1.5(6 \mathrm{mg} \times 2)$ & 0.34 \\
\hline $\mathrm{V}_{\mathrm{d}}, 1 / \mathrm{kg}$ & $3.7-5$ & 14 & $1.8-2.7$ & 2.7 \\
\hline Metabolism by cytochromes & CYP1A2 + CYP2D6 & CYP2D6 + CYP3A4 & no & CYP2D6 + CYP3A4 \\
\hline Active metabolites & 1-hydroxytacrine & 6-O-desmethyldonepezil & NAP-226-90 & $\begin{array}{l}\text { norgalantamine, } \\
\text { sanguinine }\end{array}$ \\
\hline Urinary excretion, $\%$ & $<3$ & 17 & mainly (metabolite) & 50 \\
\hline \multicolumn{5}{|l|}{ Administration } \\
\hline Dosage, mg/day & $80-160$ & $5-10$ & $6-12$ & $16-24$ \\
\hline Dose adjustment & yes & no? & yes & yes \\
\hline Number of doses per day & 4 & 1 & 2 or 3 & 2 \\
\hline Surveillance of hepatic enzymes & yes & no & no & no \\
\hline
\end{tabular}

9 The choice between the various available cholinesterase inhibitors, which cannot be based on comparative clinical trials.

10 Evaluation of an additional contribution of nicotinic stimulation, the impact of the glutamatergic approach and therefore memantine [5] and the possible combination of these various molecules. A recent clinical trial has just evaluated the donepezil/memantine combination [6].

\section{New Methods}

Considerable progress has been made in the field of Alzheimer's disease over the last two decades, in terms of diagnosis, particularly as a result of improved clinical and psychometric expertise, and treatment, with the arrival of symptomatic treatments. This progress has allowed validation of sensitive diagnostic and assessment criteria, but which must now be further improved. Progress in the basic understanding of the pathophysiology of Alzheimer's disease and the development of relevant animal models have profoundly changed our view of Alzheimer's disease and have led to the definition of different treatment objectives from those developed up until now. These objectives, to modify deterioration of the disease, to delay or even prevent its onset, inevitably lead to a review of the methodology resulting in major revisions of guidelines. In the light of current knowledge, these guidelines must be reviewed and adapted in terms of their qualitative diagnostic dimensions and their cognitive, psychological or behavioural dimensions.

One of the major barriers in the current development of new molecules is the definition of the therapeutic effect of potential drugs in Alzheimer's disease. The methodology used for clinical trials of drugs for the treatment of dementias (Alzheimer's disease, but also vascular dementias, Lewy body or Parkinson's disease) is currently very rigid (study design, assessment scales, duration, choice of patients with moderately severe impairment), leading to the validation of 'me-too' molecules. The efficacy versus placebo is tested on cognitive criteria and global clinical evaluation (Food and Drug Administration criteria), essentially based on the demonstrated effects of cholinesterase inhibitors. To our knowledge, with the possible exception of the study evaluating the effects of vitamin E, no trial has really been designed with the main hypothesis of slowing disease progression, probably due to the difficulty in defining the possible 'efficacy' in this type of protocol [7]. 


\section{Experimental Animal Models of Alzheimer's Disease}

Although the final objective of pharmacological research is the use of a molecule in man, animal experimentation is a mandatory step to generate hypotheses concerning pathophysiological mechanisms and potential pharmacological targets for Alzheimer's disease and for the preclinical evaluation of new molecules. Many animal species were used up until the middle of the 1990s (monkeys, rodents, cats, dogs or even polar bears!) with more or less approximate reproduction of neuropathological lesions and/or clinical symptoms. The situation has radically changed since 1995 with the creation of transgenic mice, following the identification of a number of gene mutations which are involved in Alzheimer's disease. Although these transgenic mice have facilitated progress in our understanding of the pathophysiological pathways involved in Alzheimer's disease, the evaluation of new pharmacological molecules is hampered by the fact that these models only reproduce some of the neuropathological lesions or only part of the cognitive impairment observed in Alzheimer's disease.

\section{Which Models for Which Lesions?}

Animal models of Alzheimer's disease can be schematically classified into two categories: models inducing lesions of the neuronal pathways responsible for clinical symptoms, particularly memory, and transgenic models, in which the animals develop some of the neuropathological lesions of Alzheimer's disease.

In lesional models, a toxic substance is injected into a cerebral region in order to destroy the neurons of a circuit involved in one of the cognitive functions altered in Alzheimer's disease. The basal nucleus of Meynert constitutes a region of choice to destroy cholinergic neurons projecting onto the hippocampus. Toxic substances leading to neuronal cell death act via the pathophysiological mechanisms involved in Alzheimer's disease: excitotoxicity for kainate or quinolinic acid, inflammation for lipopolysaccharide. Some lesional models use toxic substances that even more closely resemble the mechanisms of Alzheimer's disease, such as injection of beta-amyloid protein $(A \beta)$ into the hippocampus or administration of nerve growth factor (NGF) inhibitors. These agents can be administered by acute injection, but more frequently by chronic administration using mini-osmotic pump systems.

Many (about 50) transgenic models have been described and are able to reproduce either amyloid lesions or 'taupathic' lesions and neurofibrillary degeneration. These transgenic models, although closely reproducing pathophysiological mechanisms, are limited by the fact that they are not automatically accompanied by neuronal cell death. The prospects for the future consist of developing transgenic or knock-out animals comprising several manipulated molecular pathways (protein precursor of $A \beta$ and presenilin; tau $/ A \beta$ interaction) or animals that are genetically modified for a molecular pathway regulating the constitution of the two types of pathological lesions (knock-out mice for NGF or transgenic mice for NGF antibody, for example). Transgenic models are often based on the discovery of a gene mutation only affecting a small fraction of patients suffering from Alzheimer's disease, and these models are therefore not representative of 'common' sporadic Alzheimer's disease.

These models are usually developed and investigated in 'young' animals, while age has an important effect on the development of the lesions. This criticism is formulated in relation to a large number of experimental models, but is particularly important in the case of Alzheimer's disease because of the link between ageing and this disease. The presence of vascular risk factors also appears to play a considerable role, but is rarely taken into account in experimental models [8]. On the basis of these criticisms, the relevance of animal models of Alzheimer's disease is often questioned, especially as the investigation of these models is associated with considerable methodological problems.

\section{Which Investigations for Which Models?}

Schematically, these models can be used for three types of investigations: evaluation of neuropathological lesions, understanding of neurochemical disorders and investigation of cognitive performance. In the first case, these models provide a better characterization of the mode of development and the molecular composition of the lesions, identification of new markers and investigation of the main pathophysiological pathways. The contribution of functional genomics and proteomics should facilitate progress in this field, by identifying new pathways which are positively or negatively regulated in the course of the disease. These methods of investigation of the genome and proteome should allow to identify new markers and new pharmacological targets able to induce neuroprotection. The main difficulty concerns the integration of these numerous data into a global theory, which would allow new relevant targets to be proposed more systematically. The study of the changes in neurotransmitter concentrations, particularly dopamine and sero- 
tonin, induced by these manipulations would help to elucidate the behavioural modifications. The last level of investigation concerns evaluation of cognitive performances, especially memory functions, by appropriate tests, for example: open field, Y-maze, water maze, radial arm maze and passive avoidance. Apart from the difficulty in controlling for all factors of variability which can influence or even interfere with the investigation of behaviour, the abnormalities of cognitive functions observed during Alzheimer's disease are not always reliably reproduced in experimental models, as the methods of investigation are only able to model some types of memory disorders, while other types not evaluated in these models may be affected during Alzheimer's disease. The kinetics of onset of these disorders is also difficult to reproduce in experimental models. Further progress obviously needs to be made in the choice of the batteries of tests and the development of new paradigms, often associated with methodological difficulties. The relevance of in vitro studies of the long-term potentiation phenomenon based on these in vivo models must also be determined. Functional imaging studies could provide a better correlation between the level of the lesions and cognitive decline, with the possibility of comparing experimental and clinical data. Apart from cognitive disorders, psychological and behavioural disturbances also affect the symptomatic course of the disease. This symptomatic level is very rarely considered in models of Alzheimer's disease, although it represents a major challenge for pharmacological development.

\section{Which Models for Which Types of Pharmacological Evaluation?}

In addition to characterization of the distribution and composition of neuropathological lesions, which is essential to understand the pathophysiology of the disease, these models must also have a primarily pharmacological objective. Three types of pharmacological agents can or could be evaluated in these models: neuroprotective agents; cognition enhancers, and pharmacological agents acting on psychological and behavioural disorders.

The use of functional genomics or proteomics should generate new hypotheses in terms of neuroprotection (such as growth factors) while also testing the neuroprotective potentials of already existing drug classes, whether they are prescribed for Alzheimer's disease (nicotinic agonists, glutamatergic antagonists) or for other diseases (statins, fibrates). This preclinical type of pharmacological approach raises the problem of early detection of any acceptability problems of the molecule (see 'Alzheimer' immunization). It is important to clearly define the assessment criteria of a neuroprotective agent or a combination of neuroprotective agents in order to define the predictability of the clinical efficacy of these therapeutic strategies. This evaluation must combine morphological and functional assessments on a battery of behavioural tests, particularly memory tests.

A major challenge in the evaluation of pharmacological agents with a symptomatic action would be to extrapolate to man the results of experimental evaluations of drug combinations in relevant cognitive paradigms. The interactions between the various neurotransmitter systems involved during Alzheimer's disease prevent the possibility of massive improvement of the patient's cognitive performances without combining several pharmacological agents modulating various neurotransmission pathways. The investigation of disease models by functional imaging, under pharmacological conditions, would be very useful to make progress in this field.

\section{What Are the Prospects in Terms of Models?}

Various issues must be addressed in order to optimize the use of disease models:

1 an improved knowledge of the pathophysiology of Alzheimer's disease would allow the development of disease models in larger mammals, particularly primates, in order to refine cognitive testing,

2 a clearer definition of the relevance in man of the results obtained in transgenic mice,

3 development of new molecular investigation tools in these models,

4 improvement of cognitive and psychobehavioural investigation tools and

5 definition of guidelines to more clearly define the predictability of preclinical tests.

Although some of these issues could rapidly have practical applications, others would be more difficult to achieve and are common to all in vivo experimental models.

\section{In silico Models: The Virtual Neurochemical Brain}

These techniques, currently at the stage of development, are designed to integrate pharmacological data into a mathematical model in order to simulate the central nervous system disease, to help validate new targets and to also allow pharmacodynamic simulation in the context of drug combinations. This approach can be used to constitute a virtual synaptic cleft and modify as desired the 


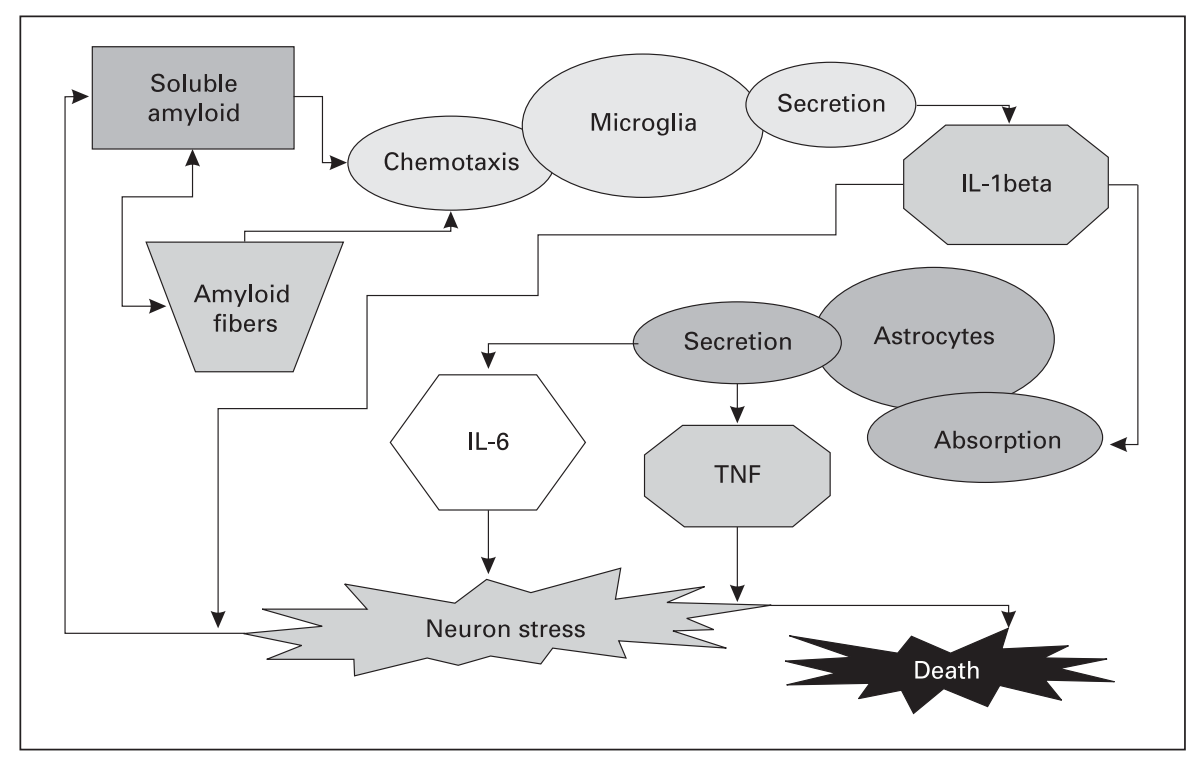

Fig. 1. Neuro-inflammation and Alzheimer's disease: scheme of the model and visualisation screen.

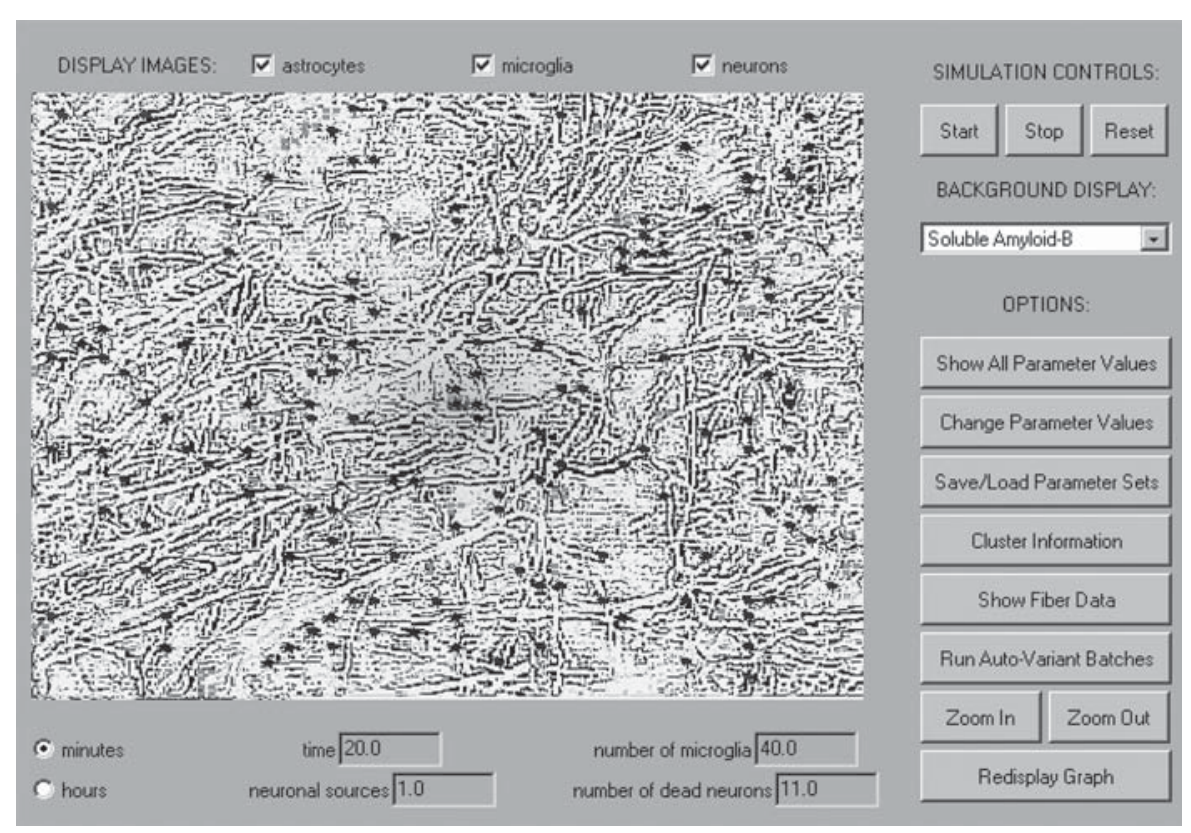

Another example concerns the neuro-inflammation observed in Alzheimer's disease. On the basis of the scheme of interrelations between neurons, astrocytes and microglial cells, as shown in figure 1, it is possible to test in silico, using a spatiotemporal model, the relative value of blocking IL-1 $\beta$ or IL-6 in order to minimize neuronal cell death induced by amyloid substance.

Other developments are able to predict the effects of drugs on the dopamine concentration, a parameter that can be easily verified experimentally. A recent study has validated in silico simulation by the results obtained ex 
vivo on striatal sections [10]. Finally, a last level of complexity allows modelling of the effect of drugs on the functioning of neuronal circuits, for example, on a model of the prefrontal cortex, tending to reproduce clinical reality in terms of attention and alertness.

\section{Clinical Investigation}

\section{At the Stage of Diagnosis}

The improvement and relative standardization of clinical examination procedures, particularly neuropsychological testing, have allowed specialized centres to achieve a diagnostic accuracy of $85-90 \%$ in Alzheimer's disease. However, our diagnostic approach still has a number of defects, especially nosographic or even conceptual, that were very well analysed by Derouesné [11]. The current diagnostic criteria for Alzheimer's disease are those of dementia, i.e. a syndrome in which cognitive disorders are so severe that they induce loss of the subject's autonomy. However, by definition, Alzheimer's disease appears and deteriorates progressively and the first clinical disorders are considered to appear at least 5-7 years before the development of true dementia. With the prospect of curative treatment, this disease must be diagnosed at an earlier stage, for example at the stage referred to as MCI. This diagnostic category, initially defined as comprising memory symptoms and pathological memory disorders, but relative sparing of other cognitive functions and the subject's autonomy, could correspond to a predementia stage of Alzheimer's disease. The supporters of this concept report that the conversion rate to Alzheimer's disease is about $12 \%$ per year. However, other teams have emphasized the serious lack of precision of the diagnostic criteria, which currently makes MCI a factor predisposing to Alzheimer's disease, rather than a real predementia syndrome. Longitudinal studies are currently underway in order to more clearly define the predementia stage of Alzheimer's disease.

\section{Biomarkers of Alzheimer's Disease}

Another approach to clinical research is designed to validate easily accessible, reproducible and specific peripheral markers of the course of the disease. The demonstration of laboratory, radiological or other markers and their evaluation as secondary endpoints (or even primary endpoints!) in the various clinical and therapeutic trials would present a number of advantages. In particular, they would allow:

The Future of Anti-Dementia Drugs
1 greater homogeneity of the patient groups studied due to their diagnostic contribution,

2 the definition of surrogate endpoints evaluating the safety and efficacy of new molecules or specific pharmacodynamic effects,

3 evaluation of the effects of these various substances in terms of pharmacogenomics, to determine or verify the proposed mechanisms of action of these various substances,

4 subsequently, the development of original study designs, possibly shorter, and less constraining for patients,

5 evaluation of the value and the effects of combination treatments in preliminary exploratory trials,

6 conduct of proof of concept validation trials by advancing from an empirical methodology to projects based on mechanistic hypotheses and

7 enhancement of the quality and safety of the clinical development of a drug to reduce its duration and even to obtain more rapid responses in terms of potential efficacy and to decrease costs.

\section{Laboratory Markers}

Among the various markers described in the literature $[12,13]$, we can try to identify those which would improve diagnostic probability. Genetic markers are only useful in the small proportion of patients with hereditary Alzheimer's disease related to gene mutations affecting amyloid precursor protein (APP) and presenilins 1 or 2 . ApoE genotyping has limited value in individual patients. At the present time, by far the most effective laboratory tool appears to be cerebrospinal fluid (CSF) assay of the tau protein and beta- 42 protein. The levels of tau protein are higher in the CSF of patients with Alzheimer's disease, but there is an excessive overlap with CSF tau levels in control subjects. However, the specificity of this type of assay could be improved by assaying the hyperphosphorylated form of tau protein. The level of beta42 protein, measured by means of a specific antibody, is decreased in patients with Alzheimer's disease compared to controls, with a sensitivity of almost $100 \%$, but a specificity of about $65 \%$. These assays may simply remain adjuvants to the diagnosis, if their specificity cannot be improved. In addition, due to the technical difficulty of these tests (lumbar puncture, requiring hospitalization, and the difficulty in performing repeated examinations), they cannot constitute a first-line examination. One of the major problems in the development of a sensitive and specific laboratory marker is that a precise correlation has not yet been established between 
molecular and neuropathological changes and the various clinical phenotypes.

\section{Imaging}

Progress in cerebral imaging [14] concerns both morphological imaging (so-called routine visual evaluation, planimetry or more specifically volumetry) and functional imaging. At the stage of diagnosis, progress could be achieved with MR volumetry. MRI allows more detailed analysis of the temporal regions than brain CT scan. In group studies, more marked temporal atrophy is observed in patients with Alzheimer's disease compared to agematched healthy subjects. However, because of the extreme variability of the lesions and the fact that other diseases can also induce temporal atrophy, the presence of these signs in a given patient is relatively non-specific. The same applies to hippocampal atrophy. On the other hand, progression of this atrophy over time appears to be more informative and can be used to distinguish between the various stages of Alzheimer's disease and between Alzheimer's disease and MCI [15]. A number of studies have shown that hippocampal volume is reduced by $12 \%$ in all early forms of Alzheimer's disease, by $20-30 \%$ in moderate forms of Alzheimer's disease and by $30-40 \%$ in severe forms. The sensitivity of these measures would be $80-100 \%$ with a specificity of $80-95 \%$. Atrophy even appears to occur before the first symptoms and the sensitivity of this technique could be increased by performing sequential volumetric measurements, for example at 6month intervals. Accentuation of hippocampal atrophy precedes the first symptoms and accelerates after onset of symptoms. The reduction of hippocampal volume appears to be correlated with the cognitive and behavioural impairment, but it particularly appears to be more sensitive to evaluate deterioration of the disease $[16,17]$. Volumetric markers could therefore constitute predictive elements and could provide a useful diagnostic contribution, but most importantly, they constitute markers or primary endpoints and no longer just surrogate endpoints (in the same way as in multiple sclerosis) to assess the effect of antineurodegenerative treatments.

Although MRI still has a limited place in the assessment of Alzheimer's disease (cost, type of examinations, reproducibility of measurements, absence of recognized normative values), volumetric markers could eventually be used to establish prognostic indices, which would have a definitive diagnostic value at the presymptomatic stage, as well as a methodological value.

Other functional imaging techniques (perfusion, diffusion or functional MRI) are currently under evaluation and could also allow early detection of alterations of target regions, according to a profile suggestive of Alzheimer's disease.

MR spectroscopy provides information about cerebral metabolism. It provides qualitative and quantitative data on cerebral distress. In Alzheimer's disease, there is a reduction of the $\mathrm{N}$-acetylaspartate/creatine ratio affecting the internal temporal lobe and an increase in myo-inositol [18] reflecting neuronal loss and a membrane lesion. These modifications occur early and are correlated with cognitive disorders. The increase in myo-inositol appears to be a constant finding in patients with MCI [19], but not in normal elderly subjects. Nevertheless, the use of these criteria in the context of individual diagnosis does not appear to be simple at the present time, as these abnormalities are relatively non-specific. The same applies to the broader context of a therapeutic trial, as data concerning the course of this parameter and its specificity are not available.

Positron emission tomography (PET) and single photon emission computed tomography (SPECT) [20] also appear to be very sensitive to demonstrate the regions of the brain affected by Alzheimer's disease and can therefore constitute an aid to the differential diagnosis. All of the various studies conducted with PET emphasize the early and constant reduction of metabolism in the parietotemporal and posterior cingulate associative cortex in Alzheimer's disease and, most importantly, these changes are observed at the presymptomatic stage. Similar findings have been reported with SPECT, on which the presence of decreased perfusion in the cingulate gyrus associated with tau protein assay in CSF allows accurate 'classification' of patients with MCI who remain at the stage of MCI and those who progress to Alzheimer's disease with a sensitivity of $88.5 \%$ and a specificity of $90 \%$ [21]. Although these studies cannot be performed as part of out-patient follow-up, they are useful in the context of development of specific drugs for Alzheimer's disease, in which diagnostic criteria are particularly important.

The development of new techniques and new markers may allow in vivo demonstration of amyloid deposits using markers such as IBOX or thioflavine derivatives. However, due the limited availability of PET scan apparatuses and the prohibitive cost of this investigation, it is unlikely to be used in routine clinical practice, but these new markers could probably be eventually developed for SPECT, which is easier to perform while possibly providing comparable results. 


\section{At the Stage of Evaluation of Treatment}

Neuropsychologists have developed increasingly sensitive and elaborate batteries of tests to assess cognitive functions at the stage of diagnosis. These tests include the Grober and Buschke test (RLI-16), the double memory test and autobiographical memory evaluation tests based on the most recent concepts of memory functioning (the course of memory with advancing age has recently been reviewed by Piolino [22]). Naming tests have also been developed for evaluation of language, such as the deno100 , specifically adapted to dementia. It is surprising that the methods of cognitive assessment used during therapeutic trials have barely advanced since the studies conducted on tacrine. The Alzheimer's Disease Assessment Scale-cognitive subscale essentially provides quantitative information and is unable to assess the deficient processes. It provides a very poor assessment of executive functions and effortful processes, which nevertheless appear to be impaired early in the course of the disease. Tests such as the Clinician Interview-Based Impression of Change Plus Caregiver Input, which combines a clinical interview with simplistic cognitive tests, deserve to be revised in order to improve their sensitivity and relevance [23]. Most of the assessment scales used in the international literature date from the 1970s and 1980s, even the Severe Impairment Battery, recently used in advanced forms of dementia.

Evaluation of the effects of treatment can no longer ignore medico-social and pharmaco-economic aspects of treatment, in terms of reduction of caregiver burden and reduction of the duration of care, either by the caregiver or by nursing staff, and delayed institutionalization. In contrast, prescription of the available molecules constitutes a major burden for the budgets of health care structures; in Belgium, a doctor independent of the prescriber evaluates the benefit provided by cholinesterase inhibitors after 6 months, using the Alzheimer's Disease Assessment Scale. Reimbursement is officially suspended if this benefit is not considered to be sufficient and treatment is stopped beyond a Mini Mental State Examination score of 12 .

Therapeutic trials have emphasized the existence of responders and non-responders. It would be relevant, from an academic point of view but also for treatment, to more clearly determine the pharmacological, genomic and clinical basis in terms of predictive factors of this response.

By limiting the definition of Alzheimer's disease to its purely cognitive aspects, we tend to neglect the psychological and behavioural aspects at the time of the diagno- sis, but also in the context of evaluation of the effects of treatment. This overcognitive view of the disease explains the delay in emphasizing the favourable effects of cholinesterase inhibitors on psychological and behavioural disorders such as apathy, delusions, hallucinations, while the beneficial effect on memory was only limited (throwing doubt on the cholinergic hypothesis). The tools used to evaluate these mental disorders are often derived from psychiatry, but tools specific to dementia should be used, such as the Neuropsychiatry Inventory, which needs to be further refined to allow a more precise evaluation of certain emotional disorders or disturbances of consciousness, which have major repercussions on the patient's family.

The definition of probable Alzheimer's disease as supposedly pure Alzheimer's disease excludes subsequent identification of cases of Alzheimer's disease decompensated by concomitant diseases, in which therapeutic intervention could be rapidly effective (mixed, vascular and degenerative forms, Lewy body forms of Alzheimer's disease). From this point of view, studies such as the SystEur study open interesting therapeutic prospects, which need to be confirmed and especially completed by a more precise analysis of the cognitive and behavioural benefits of vascular treatments (e.g. dosages, limits of risk factors).

\section{New Treatments}

Despite considerable progress, especially in the field of genetics, it must be acknowledged that no major breakthroughs have been made at the cellular and molecular levels that are likely to lead to a revision of our therapeutic strategies for Alzheimer's disease, essentially based, at the present time, on cholinergic stimulation. However, although it is somewhat utopian to imagine finding a cure for this disease, it is nevertheless true that the considerable challenges to society would justify the development of interventions designed to delay the onset of the disease or slow its course (disease modifiers) by maintaining a relative autonomy of the patients. It is now generally accepted that, as this disease is predominantly age-related, by assuming that disease-modifying strategies are more rapid than the growth of life expectancy, the number of cases would be dramatically decreased, as suggested by epidemiological data.

Consequently, without losing sight of the possibility of curative treatment, the treatment objective should now be primarily focussed on symptomatic treatments in or- 
der to improve current results and especially on the aspects of prevention/slowing of the disease process. In this context, the prospects of research can be defined according to three main axes: firstly, an approach based on cholinergic transmission to improve current symptomatic treatments; secondly, a specific approach related to the mechanisms of the lesions, essentially concerning accumulation of $\mathrm{A} \beta$ peptide and neurofibrillary formations, and finally an even more prospective approach, designed to slow the neurodegenerative process. In this last case, neuroprotection must target the potential mechanisms of cell death, which have not yet been completely elucidated in the case of Alzheimer's disease. Finally, 'repair' strategies must also be considered, but, although it is currently in the news, this approach, based on the use of transfected or non-transfected stem cells for the purposes of transplantation, remains very theoretical.

\section{Potentiation of Deficient Cholinergic Transmission}

The deficiency of cholinergic transmission in Alzheimer's disease is an established fact. Apart from tacrine, the use of new generations of cholinesterase inhibitors gives satisfactory results, particularly at the early stages of the disease, without any major adverse effects. The second strategy designed to stimulate cholinergic transmission is based on the potential administration of cholinergic receptor agonists [24-27]. Several muscarinic receptor agonists have been tested, such as arecoline or xanomeline, with varying degrees of success. In this field, the development of nicotinic agonists respecting the structural specificity of receptors mainly present in cortical and hippocampal structures (especially forms rich in $\alpha_{4}$ and $\alpha_{7}$ subunits) could constitute an interesting therapeutic approach. This line of research is developed by some pharmaceutical companies, but the approach to this problem nevertheless appears somewhat simplistic, particularly in relation to the complexity of cortical and hippocampal neuronal lesions, which are not simply limited to cholinergic degeneration. Furthermore, the specific distribution of cholinergic circuits to many cerebral regions will have repercussions on other neurotransmitters and will be reflected by clinical effects, such as those observed during the use of cholinesterase inhibitors.

\section{Limit the Lesions of Alzheimer's Disease}

Three main types of lesions are observed, but their specificity in relation to Alzheimer's disease and their correlation with behavioural disorders and cognitive impairment have obviously not been clearly demonstrated: the formation of senile plaques predominantly composed of A $\beta$ peptide; the formation of neurofibrillary degeneration, in which the tau protein is predominant, and inflammatory lesions with the development of a glial reaction composed of proliferation of microglia and hypertrophy of certain astrocytes. At the present time, attempts to limit these lesions are essentially focussed on $\mathrm{A} \beta$ peptide with 3 major objectives: limit the synthesis of the peptide; limit its aggregation, and 'dissolve' these aggregates.

Progress in our understanding of the metabolism of $A \beta$ protein and its precursor, APP, has led to the development of strategies designed to block the pathological metabolic pathway leading to the formation of $A \beta$ peptide, involving $\beta$-secretases ( $\beta$-site APP cleaving enzyme) or $\gamma$-secretases (whose activity is modulated by presenilins), versus the 'normal' pathway using $\alpha$-secretases, regulated by protein kinase $C$. Four major strategies have been proposed to prevent the formation and accumulation of $A \beta$ peptide [28-30].

1 Immunization with a synthetic $A \beta$ peptide, which continues to be a line of research despite the various difficulties encountered, especially in clinical trials. The toxicity of $A \beta$ peptide, especially in relation to synapses (synaptosis), can be explained by soluble intermediate oligomers, which now appear to be the preferential target of therapeutic antibodies [31]. This extremely promising line of research in transgenic mice overexpressing $\mathrm{A} \beta$ protein (memory disorders, evaluated in the water maze, were improved by the vaccine) may not be completely abandoned if the risk of encephalitis can be controlled [32].

2 The use of $\beta$-sheet breaker peptide analogues, which bind to $A \beta$ to reduce its aggregation, as the $\beta$-sheet structure contributes to make the $A \beta$ protein insoluble.

3 Pharmacological inhibition of $\gamma$-secretases.

4 Modulation of the activity of presenilins.

Apart from immunization, pharmacological inhibition of $\beta$ - and $\gamma$-secretases is also under development, for example by using certain peptides derived from calpains, cathepsins or non-peptide derivatives that are in the process of being identified. It should also be noted that a reduction of the formation of $A \beta$ peptide can be obtained by modulating the activity of $\alpha$-secretases of the 'normal' 
pathway leading to the formation of a soluble protein, especially by stimulation of $\mathrm{mGluR}_{1}$ (linked to stimulation of the PLC pathway), excitatory amino acid (EAA) or $\mathrm{M}_{1}$ or $\mathrm{M}_{3}$ muscarinic receptors.

Finally, in relation to the potential limitation of aggregation of $A \beta$ peptide, Bush [33] reported that, in vitro, copper and zinc are two elements that promote aggregation related to the formation of senile plaques. In view of the increased cerebral concentrations of these two metals with age, it appears logical that limitation of copper and zinc concentrations in the brain could help to limit the formation of $\mathrm{A} \beta$ peptide deposits and subsequent plaque formation. This observation is the basis for apparently successful attempts to reduce amyloid deposits in transgenic mice overexpressing APP, as well as other trials whose results have not yet been published, involving clioquinol, an antibiotic withdrawn from the market following cases of subacute myelo-optic neuropathy, but known to bind copper and zinc and decrease their cerebral concentrations in Alzheimer's disease [34]. Other metal chelators, such as deferoxamine, would have similar properties. Although it is difficult to draw any definitive conclusions, this strategy deserves further investigation, as it appears to be a simple and inexpensive way to influence abnormal APP metabolism. It is interesting that encouraging results on solubilization of the prion protein have been obtained with quinacrine, but have already been invalidated in clinical trials.

No significant development in terms of therapeutic progress has been made in the field of the tau protein and neurofilaments, possibly more directly related to neuronal cell death in Alzheimer's disease. Hyperphosphorylation of the tau protein is one possible approach involving protein kinases [35] and tau proteins could be influenced by actions on the cytoskeleton, possibly by means of cytochalasins. In this context, various protein kinase inhibitors, such as staurosporin, or agents acting indirectly on phosphorylation, such as lithium or benzimidazoles, could present a therapeutic value which remains to be evaluated. Research is currently underway to discover specific inhibitors of cdk5 and GSK3A [36].

Finally, in the field of lesions, the inflammatory process that accompanies plaque formation can be modulated by non-steroidal anti-inflammatory drugs. Consequently, in addition to microglial proliferation, some authors have considered Alzheimer's disease to resemble a state of 'chronic' inflammation, marked by high levels of cytokine production, especially IL-1 and IL-6. Although a real therapeutic action cannot be evaluated at the present time, it is possible that aspirin, ibuprofen or indo- methacin could have beneficial effects to 'slow' the pathological process, as proposed by some authors. Interestingly, the 'protective' action of these anti-inflammatory drugs could interfere with the cell death process, for example via cyclo-oxygenase inhibition (Cox-2 inhibitors) or by an increase in the Bax factor, key steps in the apoptotic process. The finding that anti-inflammatory treatments would be useful in the prevention of Alzheimer's disease, but not in patients with diagnosed Alzheimer's disease, suggests that neuro-inflammatory disease would play an important role at the presymptomatic stage [37].

\section{Towards Effective Neuroprotection?}

Strictly speaking, the field of neuroprotection extends beyond the field of Alzheimer's disease, but it clearly concerns both acute degenerative diseases, such as stroke, as well as neurodegenerative diseases. It would therefore be useful to study the mechanisms of neuronal degeneration in order to try to limit the effects of this process. This is a rapidly expanding field of research and the main pathways investigated have recently been described in the literature. In the context of Alzheimer's disease, the development of ways of prolonging the asymptomatic stage of the disease or slowing progression in symptomatic patients in order to prolong their autonomy would be of major benefit to society. However, the mechanisms of neuronal degeneration have not yet been elucidated, although it is generally accepted, probably somewhat excessively, that apoptosis plays a major role in this process.

\section{Replacement Strategies}

Although, once again, it is difficult to speak of prevention of Alzheimer's disease, it is nevertheless accepted that certain replacement strategies could contribute to reduce the incidence of the disease. First of all, a conventional approach consists of oestrogens and more generally certain steroids or derivatives, such as DHEA. There is an intense controversy in this field concerning the real beneficial effects and disadvantages of this type of treatment. Hormone replacement therapy (HRT) for postmenopausal women was originally considered to be able to decrease the age-related risk of Alzheimer's disease (possibly by delaying the process?) or slow progression of the disease in demented patients, but Smith et al. [38] showed that HRT could be an aggravating factor. These limited observations could possibly be related to the trophic effects of these hormones related to development or 
characterized in vitro in cell models. However, it is unlikely that neuroprotection could have a specific action in the context of Alzheimer's disease.

Similarly, administration of cocktails of antioxidants (such as vitamin $\mathrm{A}$ - retinol, vitamin $\mathrm{C}$ - ascorbic acid, vitamin $\mathrm{E}-\alpha$-tocopherol, coenzyme $\mathrm{Q}$ or Ginkgo biloba extracts), but also melatonin or SOD mimetics and other radical oxygen species scavengers, is designed to reduce the harmful effects of free radicals, based on the hypothesis of the role played by certain forms of oxidative stress in neuronal cell death. This hypothesis is currently very popular in the context of neuroprotection, however more in relation to other neurodegenerative diseases, such as Parkinson's disease, than Alzheimer's disease. In the context of the possible effects of antioxidants, we should also note the potential action of selegiline, a type B monoamine oxidase inhibitor that has also been described to exert neurotrophic effects.

Other proposed replacement strategies use Ginkgo bi$l o b a$, but, in this context, for its potential effects on membrane fluidity, which facilitates intercellular communication, or even certain approaches designed to reduce the high levels of homocysteine, which appear to be a risk factor for Alzheimer's disease, especially by vitamin $\mathrm{B}$ supplements $\left(\mathrm{B}_{6}, \mathrm{~B}_{12}\right)$, promoting the metabolism of excess homocysteine.

Finally, the cholesterol-lowering effects of statins are considered to be associated with a reduction of the agerelated risk of Alzheimer's disease. The most likely hypothesis for this effect is that of an increased risk of Alzheimer's disease in patients with hypercholesterolaemia, linked to the risk of cardiovascular disease, which also appears to increase the risk of dementia.

\section{Neuroprotection against Excitotoxicity}

This approach consists of developing objective ways to limit a mechanism of neuronal cell death likely to result from excessive excitatory neurotransmission, involving EAAs, especially glutamate, major neurotransmitters of the central nervous system and therefore act on Alzheimer's disease [39]. This type of degenerative process has been implicated in several neurodegenerative diseases, such as amyotrophic lateral sclerosis (ALS), Huntington's disease, certain aspects of Parkinson's disease and in many ischaemic, anoxic or post-traumatic processes. The role of EAAs can be considered to be either causal or reactive. Although, in some cases, a disturbance of the glutamatergic synapse is considered to be directly responsible for cytotoxic levels of extracellular glutamate, it has also been demonstrated that, in other situations, an ex- cess of extracellular glutamate is the result of a more general deficit of cellular and especially membrane metabolism, for example in the context of anoxic processes.

As the neurons that degenerate in Alzheimer's disease are mainly hippocampal and cortical neurons, i.e. mainly glutamatergic neurons, the essential question is not whether or not excitotoxicity plays a real role in Alzheimer's disease, but to determine why these neurons present a particular susceptibility to the degenerative process and the ways in which they can be protected from this process.

The answer to these questions is very complex. Theoretically, the most effective neuroprotection strategies are not necessarily the most sophisticated, although it appears utopian to try to apply them in the context of treatment of Alzheimer's disease. The most promising approaches, at the present time, are based on reduction of the excitation process, for example by promoting GABAergic inhibitory neurotransmission. An illustration of this approach is the potential value of direct or indirect GABAergic agonists, such as propofol, valproic acid or felbamate, but which would be more effective in acute diseases, and riluzole that acts as a glutamatergic inhibitor, globally reducing glutamatergic transmission.

Any discussion of neuroprotection must also include EAA receptor antagonists, targeting NMDA ionotropic receptors or non-NMDA ionotropic receptors. In this context, apart from the problems associated with the use of MK 801 and ketamine, the recent introduction of memantine as a treatment for severe forms of Alzheimer's disease due to its non-competitive NMDA receptor antagonist action raises the question of its real neuroprotective efficacy and the benefit obtained, while also raising the issue of a possible deterioration of the deficit of NMDA transmission related to the disease and its consequences on synaptic plasticity in terms of memorization related to long-term potentiation. The same applies to the use of AMPAkines, modulators of non-NMDA receptors.

The recent demonstration of the potential neuroprotective effects of metabotropic glutamate receptor $\mathrm{mGluR}_{5}$ antagonists or, on the contrary, glutamate receptor $\mathrm{mGluR}_{2 / 3}$ agonists appears to open up new therapeutic strategies in Alzheimer's disease. These pharmacological agents targeted to $\mathrm{mGluR}$ receptors have been found to be effective in animal models and in vitro, but it is clear that these models do not correspond to the pathological processes of Alzheimer's disease. The effects induced by stimulation of $\mathrm{mGluR}_{2 / 3}$ receptors could be indirect, resulting from increased production of growth factors by 
astrocytes. In any case, the role of EAA metabotropic receptors in neuroprotection is a very promising line of research that deserves thorough investigation.

Before discussing the innovative field of the role of astrocytes and glutamate transporters in the neurodegenerative process, we should mention two lines of research related to the cytotoxicity of EAAs: one line of research is investigating ionized calcium as an effector of neuronal cell death by apoptosis and the other concerns a possible endogenous neuroprotective action of adenosine acting as an EAA 'cotransmitter', at least in some neuronal systems. In the first case, the use of calcium antagonists, such as nimodipine, has been recommended for a long time. However, in the context of Alzheimer's disease, this strategy does not appear to have given any major results. In the current state of knowledge, EAA receptors, mainly NMDA receptors, are the only receptors whose functioning results in massive calcium influx into the cell. Limiting the intracellular effects of calcium therefore corresponds to limiting the action of NMDA receptors, especially when they are overactive.

The neurobiological basis for the role of adenosine is as follows: in some neuronal systems using EAA such as neurotransmitter, it is generally accepted that neurons actually corelease both glutamate and adenosine. In this context, adenosine is considered to be an agent limiting the effects of glutamate, by acting on at least two levels: at the presynaptic level by limiting the release of glutamate via a mechanism involving presynaptic receptors and at the postsynaptic level by antagonizing the effects of stimulation of EAA receptors by specific type $A_{1} / A_{2}$ purinergic receptors. Limiting the synaptic elimination of adenosine by pharmacologically blocking its reuptake or stimulating purinergic receptors corresponds to promoting this endogenous inhibitory mechanism of glutamatergic transmission. Some encouraging in vitro results have been reported, but this hypothesis still needs to be tested in situations more closely resembling neurodegenerative processes.

In the field of excitotoxicity, data are currently converging to make EAA transports involved in the synaptic inactivation of neurotransmitters a potential target for neuroprotective agents. The studies by Rothstein et al. [40] were the first to suggest a defect of inactivation by reuptake in the degenerative process related to certain forms of ALS, following the identification and cloning of the main subtypes of transporters (the three main subtypes are called $\mathrm{EAAT}_{1}, \mathrm{EAAT}_{2}$ and $\mathrm{EAAT}_{3}$ ). Endogenous glutamate now clearly appears to have a cytotoxic action [41]. A defect of elimination of EAA can therefore contribute to neurodegenerative processes. However, in the current state of knowledge, there is a major obstacle to this proposal: it is pharmacologically possible to block EAA transporters, but they cannot be stimulated. Studies on the glutamatergic synapse have recently led to the proposal of a new mechanism of cell death possibly resulting from a defect of inactivation by reuptake of EAA [42]. Investigation of the cellular localization of EAA transporters has demonstrated that the various transporter subtypes each have a more or less specific localization and tissue expression. The EAAT E $_{1}$ transporter (also called GLAST) is mainly located in cerebellar astrocytes rather than neurons. The EAAT ${ }_{2}$ transporter (GLT1) is also located in astrocytes, but presents a large cerebral distribution, especially in the cortex and hippocampus. Finally, the $\mathrm{EAAT}_{3}$ transporter $\left(\mathrm{EAAC}_{1}\right)$ is, on the contrary, located on neurons and is expressed by postsynaptic neurons at central glutamatergic synapses (fig. 2). The results of a whole series of in vitro studies conducted on astrocyte cultures clearly show that inhibition of EAA transporters induces astrocyte death via a mechanism independent of excitotoxicity, corresponding to an oxidative process involving free radicals [43].

\section{Neuroprotection and Growth Factors}

In theory, the field of growth factors is extremely promising to limit neurodegenerative processes. Most recent discoveries show a certain specificity of the main families of these factors in relation to phenotypically identified neuronal populations, such as the action of brain-derived neurotrophic factor or certain neurotrophins in relation to dopaminergic neurons or even NGF in relation to cholinergic neurons, for example. This field is obviously more complex than initially imagined and identification of these growth factors is currently underway, with particular attention to characterization of the receptor subtypes related to these various factors. The idea of promoting neurotrophic effects by providing neurons with the growth factors that are possibly deficient should therefore prove to be an effective strategy to 'salvage' certain populations susceptible to either one of these neurodegenerative processes. Theoretically, however, this approach is limited by an obvious obstacle: the peptide nature of these growth factors is not compatible with systemic administration. Research scientists are therefore trying to produce nonpeptide analogues that can be administered to patients (and which are more stable in relation to the action of peptidases) and are also trying to develop 'vectors' allowing access of peptide factors to the central nervous system. This field of research is very active [44], but the results to 


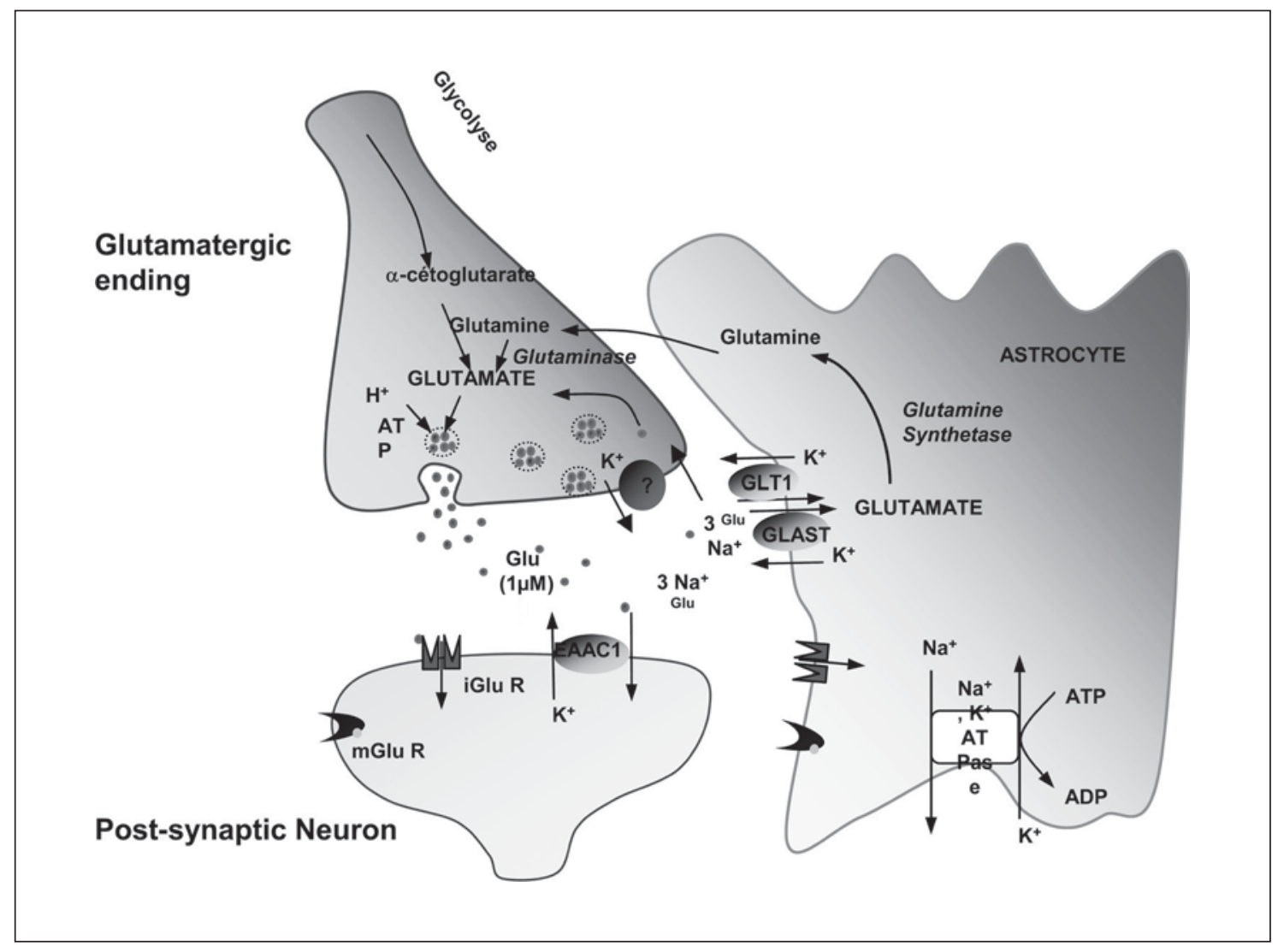

Fig. 2. The glutamatergic synapse (Scheme LNCF CNRS, Marseille, France). Glu = Glutamate; iGluR = ionotropic glutamate receptor; $\mathrm{mGluR}=$ metabotropic glutamate receptor; GLT1 = glutamate transporter type 1; GLAST = glutamate/aspartate transporter.

date appear to be very disappointing, despite the discovery of several interesting molecules, such as TrKB receptor agonists, or glial cell line-derived neurotrophic factor analogues, such as neurturin or the potential use of cells that are transfected in vitro and secondarily transplanted and able to produce growth factors as required.

An alternative strategy therefore consists of indirectly promoting the production of growth factors from cells that naturally synthesize these factors in the nervous system, especially astrocytes. Pharmacological stimulation of certain EAA-type mGluR receptors, described above, is one of the strategies based on this approach. Similarly, AMPAkines could have similar effects, in the same way as certain neuropeptides or their analogues, such as VIP, which stimulates the production of various growth factors, or even possibly and more surprisingly, lithium. This field of research, which once again places glial cells and especially astrocytes, but also endothelial cells, at the centre of the process, is certainly an interesting approach, but it is only in the very early stages and progress in this field should be followed very closely.

\section{Cell Therapy and Gene Therapy}

This very topical field of research must be discussed, if only briefly. In the current state of research, two strategies have been proposed. The first method, as suggested above, consists of using in vitro transfected cells, possibly immortalized to continually produce peptide factors or proteins which could compensate for the deficient endogenous biosynthesis. This is the case for growth factors, for example, but this strategy could also include the production of antibodies able to block the action of certain deleterious factors. For example, in an animal model, it has recently been possible to block the action of an endogenous inhibitory factor of nerve regeneration in the motor system after a lesion, a myelin-inhibitory factor, by trans- 
planting transfected cells secreting antibodies that neutralize the action of this factor. This field should continue to develop in view of the widespread use of gene transfection techniques, but it raises the question of the nature and the origin of the cells used for transfection and transplantation, as well as the problems of their potential rejection and the risks related to the transfection process, which generally uses viruses.

A second strategy has been proposed more recently, using stem cells. This approach is also based on two types of strategies. The first one proposed is designed to use embryonic stem cells, which, once the process has been fully mastered, could eventually be differentiated in vitro prior to implantation, as ad hoc cells. However, this strategy also raises the problem of the origin of the cells and the associated immune process. A second strategy would consist of using 'adult' embryonic cells, ideally taken from the patient himself, differentiated to form the missing neurons and subsequently transplanted into the same patient. Maybe one day, it will be possible to promote cell division, differentiation and migration in situ. In the cases of Alzheimer's disease, without claiming to be able to replace all of the cortical neurons, it is nevertheless reasonable to imagine stimulation of neurogenesis in the hippocampus where the mechanisms are starting to be elucidated, which would have beneficial effects on certain cognitive functions, at least in the early stages of the disease [45].

\section{Most Promising Lines of Research}

This review of the possibilities of promoting new strategies for the treatment of Alzheimer's disease does not pretend to be exhaustive, but is simply designed to highlight several lines of research that have developed as a result of progress of our knowledge in the fields of neurobiology and molecular pharmacology. Consequently, other approaches not discussed above are not considered to be less important, but their potential use appears to be less specific at the present time, e.g. intervention on the signalling pathways that direct cell death and the apoptotic process, which could usefully adopt caspase inhibitors [46]. A surprising example in this context is the resurrection of an old tetracycline, minocycline, an antibiotic considered to be an inhibitor of apoptosis, by inhibiting the release of cytochrome c [47], which is currently under investigation in Huntington's disease and ALS [48]. Other possible interventions could be designed to stimulate the cAMP pathway, especially by using phos- phodiesterase inhibitors or by using factors with trophic effects such as EPO, or more indirect interventions designed to stimulate behavioural effects, such as stimulation of the dopaminergic transmission involved in the regulation of cognitive functions and attentional processes, or serotoninergic stimulation, which could play a central role in neuroplasticity and stimulation of secondary neurogenesis in adults.

Many lines of research are therefore underway, but, at the moment, they simply remain lines of research and not yet therapeutic strategies, with the notable exception of cholinergic stimulation. A better understanding of the basic mechanisms underlying cerebral functioning and the pathological processes responsible for Alzheimer's disease would therefore provide us with the means to propose more effective management of patients, probably based on effective delay of the expression of the disease, if it cannot be eradicated [49].

\section{Conclusion}

Ethically, the announcement of a diagnosis of Alzheimer's disease implies that a treatment must be proposed. Similarly, any financial commitment to basic or clinical research should only be allocated when the main lines of this research are clearly justified. The present review demonstrates that these two postulates are real despite a certain degree of dissatisfaction in relation to current treatments and especially a considerable perplexity concerning the multitude of potential biochemical targets for the drugs of tomorrow. The essential issue at the present time is to accelerate the innovation process by forming a multidisciplinary alliance around and against this scourge. The objectives of such an alliance are clear and threefold: restore the patient's intellectual functions, delay or even prevent institutionalization, help to optimize and humanize the last decades of life.

Operatively or pragmatically, dichotomous discussions must be a thing of the past (for example: symptomatic versus cytoprotection; tau versus amyloid) and every effort must be made to evaluate all hypotheses, however varied. A multidisciplinary approach, ranging from research on the neuron (or synapse) to social (or populational) issues, should allow progress in the pharmacology of Alzheimer's disease and therefore its treatment. The ideal treatment could be a combination of molecules directed against several targets or a molecule with several valences. 
Philosophically, the current disappointment of pharmacologists can be formulated by the observation that all drugs presently in use or under evaluation in Alzheimer's disease are old molecules: e.g. tacrine, metrifonate, memantine, clioquinol or minocycline. A change of direction in pharmacological innovation can only be achieved by totally revising the principles of the philosophy governing current research, largely inspired by the logical atomism of Wittgenstein with a risk of a fragmented or partial vision of reality. If only the mythical story of Pyg- malion could repeat itself in pharmacological research: 'By dint of loving care and desire, the statue came to life in his arms' [50].

\section{Acknowledgments}

The authors would like to thank Laboratoires Janssen-Cilag for their assistance in the preparation of this seminar and PR International for their logistic support.

\section{References}

1 Citron M: Strategies for disease modification in Alzheimer's disease. Nat Rev Neurosci 2004;5:677-685.

$\checkmark 2$ Michel BF, Estadieu MC, Gueriot C, Berthezene $\mathrm{P}$, Allain $\mathrm{H}$, Bongrand $\mathrm{MC}$, Bonnefoy B, Bourrin JC, Chaix L, Graa K, Lejeune A, Messana M, Pras P, Ribiere J, Rihet P, TimonDavid P, Tintignac A, Vincent S, Verdier JM, Gastaut JL: Traitement de la maladie d'Alzheimer au long cours: à propos d'une cohorte de 255 patients traités quatre ans par tacrine. Rev Neurol (Paris) 2001;11:13651375.

3 Bentué-Ferrer D, Michel B-F, Reymann J-M, Allain H: Les médicaments face à la maladie d'Alzheimer. Revue Gériatr 2001;26:511522.

$\checkmark 4$ Allain H, Bentué-Ferrer D, Tribut O, Belliard S: Drug therapy of fronto-temporal dementia. Hum Psychopharmacol 2003;18:221-225.

$\checkmark 5$ Reisberg B, Doody R, Stoffler A, Schmitt F, Ferris S, Mobius HJ; Memantine Study Group: Memantine in moderate-to-severe Alzheimer's disease. N Engl J Med 2003;348:1333-1341.

-6 Tariot P-N, Farlow M-R, Grossberg G-T, Graham S-M, Mcdonald S, Gergel I: Memantine treatment in patients with moderate to severe Alzheimer disease already receiving donepezil: A randomized controlled trial. JAMA 2004; 291:317-324.

7 Lebert M: Slowing the progression of Alzheimer's disease: Methodologic issues. Alzheimer Dis Assoc Disord 1999;11(suppl 5):510-521.

$>8$ Allain H, Bentué-Ferrer D, Tribut O, Pinel J-F: Drugs and vascular dementia. Dement Geriatr Cogn Disord 2003; 16:1-6.

9 Lazarewicz M, Spiros A, Finkel L, Carr R, Geerts H: Using a computer simulation of the synapse to understand nicotinic receptor modulation by galantamine. 8th Int Alzheimer Congr, Stockholm, 2002.

10 Geerts H, Lazarewicz M, Spiros A, Finkel L, Carr R: Galantamine benefits in Alzheimer's disease are related to increases in dopamine output. Schizophr Res 2003;60(suppl 1):135 (abstr).
11 Derouesné C: Qu'est ce que la démence? 2. Un concept flou. Psychol Neuropsychiatr Vieil 2003;1:15-24.

12 Teunissen C-E, De Vente J, Steinbusch H-WM, De Bruijn C: Biochemical markers related to Alzheimer's dementia in serum and cerebrospinal fluid. Neurobiol Aging 2002;23:485508.

13 Pratico D, Clark C-M, Lee V-M, Trojanowski J-Q, Rokach J, Fitzgerald G-A: Increased 8,12iso-iPF2alpha-VI in Alzheimer's disease: Correlation of a noninvasive index of lipid peroxidation with disease severity. Ann Neurol 2000;48:809-812.

14 Wolf H, Jelic V, Gertz H-J, Nordberg A, Julin P, Wahlund L-O: A critical discussion of the role of neuroimaging in mild cognitive impairment. Acta Neurol Scand 2003(suppl);179:52 76.

15 Fox N-C, Freeborough P-A: Brain atrophy progression measured from registered serial MRI: Validation and application to Alzheimer's disease. J Magn Reson Imaging 1997; 7:10691075 .

16 Fox NC, Cousens S, Scahill R, Harvey RJ, Rossor $\mathrm{MN}$ : Using serial registered brain magnetic resonance imaging to measure disease progression in Alzheimer disease. Arch Neurol 2000; 57:339-443.

17 Jack CR Jr, Slomkowski M, Gracon S, Hoover TM, Felmlee JP, Stewart K, Xu Y, Shiung M, O'Brien PC, Cha R, Knopman D, Petersen $\mathrm{RC}$ : MRI as a biomarker of disease progression in a therapeutic trial of milameline for $\mathrm{Alz}$ heimer's disease. Neurology 2003;60:253260.

18 Valenzuela M-J, Sachdev P: Magnetic resonance spectroscopy in Alzheimer's disease. Neurology 2001;56:592-598.

19 Catani M, Cherubini A, Howard R, Tarducci R, Pelliccioli GP, Piccirilli M, Gobbi G, Senin U, Mecocci P: (1)H-MR spectroscopy differentiates mild cognitive impairment from normal aging. Neuroreport 2001;12:2315-2317.

20 Petrella J-R, Coleman R-E, Doraiswamy P-M: Neuroimaging and early diagnosis of Alzheimer disease: A look of future. Radiology 2003; 226:315-336.
-21 Okamura N, Arai H, Maruyama M, Higuchi M, Matsui T, Tanji H, Seki T, Hirai H, Chiba H, Itoh M, Sasaki H: Combined analysis of CSF tau levels and [(131)I] Iodoamphetamine SPECT in mild cognitive impairment: Implications for a novel predictor of Alzheimer's disease. Am J Psychiatry 2002;159:474-476.

22 Piolino P: Le vieillissement normal de la mémoire autobiographique. Psychol Neuropsychiatr Vieil 2003;1:25-35.

23 Quinn J, Moore M, Benson D-F, Clark R, Doody R, Jagust W, Knopman D, Kaye J-A: A videotaped CIBIC for dementia patients. Validity and reliability in a simulated clinical trial. Neurology 2002;58:433-437.

24 Sramek J-J, Cutler N-R: Recent developments in the drug treatment of Alzheimer's disease. Drug Aging 1999;14:359-373.

$>25$ Emilien G, Beyreuther K, Masters C, Maloteaux J-M: Prospects for pharmacological intervention in Alzheimer's disease. Arch Neurol 2000;57:454-459.

26 Cutler N-R, Sramek J-J: Review of the next generation of Alzheimer's disease therapeutics: Challenges for drug development. Prog Neuropsychopharmacol Biol Psychiatry 2001;25:2757.

27 Allain H, Bentué-Ferrer D, Tribut O, Gauthier S, Michel B-F, Drieu-La Rochelle C: Alzheimer's disease: The pharmacological pathway. Fundam Clin Pharmacol 2003;17:419-428.

28 Scorer CA: Preclinical and clinical challenges in the development of disease-modifying therapies for Alzheimer's disease. Drug Discov Today 2001;6:1207-1219.

$>29$ Citron M: Emerging Alzheimer's disease therapies: Inhibition of $\beta$-secretase. Neurobiol Aging 2002;23:1017-1022.

30 Gandy S: Molecular basis for anti-amyloïd therapy in the prevention and treatment of Alzheimer's disease. Neurobiol Aging 2002;23: 1009-1016.

-31 Kayed R, Head E, Thompson J-L, McIntire TM, Milton SC, Cotman CW, Glabe CG: Common structure of soluble amyloid oligomers implies common mechanisms of pathogenesis. Science 2003;300:486-488. 
-32 Hock C, Konietzko U, Streffer J-R, Tracy J, Signorell A, Muller-Tillmanns B, Lemke U, Henke K, Moritz E, Garcia E, Wollmer MA, Umbricht D, de Quervain DJ, Hofmann M, Maddalena A, Papassotiropoulos A, Nitsch RM: Antibodies against beta-amyloid slow cognitive decline in Alzheimer's disease. Neuron 2003; 38:547-554.

33 Bush A-I: Metal complexing agents as therapies for Alzheimer's disease. Neurobiol Aging 2002;23:1031-1038.

-34 Regland B, Lehmann W, Abedini I, Blennow K, Jonsson M, Karlsson I, Sjogren M, Wallin A, Xilinas M, Gottfries CG: Treatment of Alzheimer's disease with clioquinol. Dement Geriatr Cogn Disord 2001;12:408-414.

$>35$ Castro A, Martinez A: Inhibition of tau phosphorylation: A new therapeutic strategy for the treatment of Alzheimer's disease and other neurodegenerative disorders. Expert Opin Ther Patents 2000;10:1519-1527.

$\checkmark 36$ De Strooper B, Woodgett J: Mental plaque removal. Nature 2003;423:392-393.

>37 McGeer P-L, McGeer E-G: Local neuroinflammation and the progression of Alzheimer's disease. J Neurovirol 2002;8:529-538.

>38 Smith M-A, Perry G, Atwood C-S, Bowen R-L: Estrogen replacement and risk of Alzheimer disease. JAMA 2003;289:1100.
39 Nieoullon A: Bases cellulaires des processus neurodégénératifs. Thérapie 1998;53:21-29.

40 Rothstein J-D, Dykes-Hoberg, Pardo C-A, Bristol L-A, Jin L, Kuncl Rw, Kanai Y, Hediger M-A, Wang Y, Schielke J-P, Weltry F-D: Knock out of glutamate transporters reveals a major role for astroglial transport in excitotoxicity and clearance of glutamate. Neuron 1996; 16:675-686.

41 Lievens J-C, Bernal F, Forni C, Mahy N, Kerkerian-Le Goff L: Characterization of striatal lesions produced by glutamate uptake alteration: Cell death, reactive gliosis, and changes in GLT1 and GADD45 mRNA expression. Glia 2000;29:222-232.

42 Had-Aissouni L, Re D-B, Nieoullon A, Kerkerian-Le Goff L: Importance of astrocytic inactivation of synaptically released glutamate for cell survival in the central nervous system Are astrocytes vulnerable to low intracellular glutamate concentrations? J Physiol (Paris) 2002;96:317-322.

43 Re D, Boucraut J, Samuel D, Birman S, Kerkerian-Le Goff L, Had-Aissouni L: Glutamate transport alteration triggers differentiationstate selective oxidative death of cultured astrocytes: A mechanism different from excitotoxicity depending on intracellular GSH contents. J Neurochem 2003;85:1159-1170.
44 Lesh K-P: Gene transfert to the brain: Emerging therapeutic strategy in psychiatry? Biol Psychiatry 2002;45:247-253.

45 Zhao M, Momma S, Delfani K, Carlen M, Cassidy RM, Johansson CB, Brismar H, Shupliakov O, Frisen J, Janson AM: Evidence for neurogenesis in the adult mammalian substantia nigra. Proc Natl Acad Sci USA 2003;100: 7925-7930.

46 Friedlander RM: Mechanisms of disease: Apoptosis and caspases in neurodegenerative diseases. N Engl J Med 2003;348:1365-1375.

47 Zhu S, Stavrovskaya IG, Drozda M, Kim BY, Ona V, Li M, Sarang S, Liu AS, Hartley DM, Wu du C, Gullans S, Ferrante RJ, Przedborski S, Kristal BS, Friedlander RM: Minocycline inhibits cytochrome $\mathrm{c}$ release and delays progression of amyotrophic lateral sclerosis in mice. Nature 2002;417:74-78.

48 Li M, Ona VO, Guegan C, Chen M, JacksonLewis V, Andrews LJ, Olszewski AJ, Stieg PE, Lee JP, Przedborski S, Friedlander RM: Functional role of caspase 1 and caspase 3 in ALS transgenic mouse model. Science 2000;288: 335-339.

49 Mattson MP: Pathways towards and away from Alzheimer's disease. Nature 2004;430: 631-639.

50 Lebrun C: Le futur comme objet de désir. Futuribles 2003;286:29-42. 